\title{
Emotions and Video Sharing Behavior on Facebook of Young Generation
}

\author{
DINH Tien Minh ${ }^{1}$, LE Thi Hue Linh², NGUYEN Ngoc Lan Huong ${ }^{1}$ \\ ${ }^{1}$ Division of Marketing, School of International Business - Marketing, \\ University of Economics Ho Chi Minh City, Ho Chi Minh, Vietnam \\ ${ }^{2}$ Department of Marketing, Faculty of Business Administration, \\ Ho Chi Minh City Open University, Ho Chi Minh, Vietnam \\ *Corresponding author: dinhtienminh@ueh.edu.vn
}

\begin{abstract}
\section{Research purpose:}

This paper aims at exploring the influence of emotions on the video-sharing behavior on Facebook of the young generation in a developing country.

\section{Research motivation:}

Video is much more attractive than text on the Facebook platform and the young people are likely to watch and share the video that they love and get emotional both negative and positive. The research is mainly conducted based on this trend and contributes to the behavior studies in the world.

\section{Research design, approach, and method:}

The research applies both qualitative and quantitative research to examine the reliability of the design research model. Survey data were gathered from a sample of 528 respondents. Structural equation modeling was used to test the hypothesized relationships.

\section{Main findings:}

Findings revealed that strong positive emotions (joy, surprise), strong negative emotions (anger, disgust, fear), and weak negative emotions (sadness) all had a positive effect on video sharing behavior on Facebook of the young generation. The group of strong positive emotions and strong negative emotions strongly influenced video-sharing behavior more than the group of weak negative emotions.
\end{abstract}

\section{Practical/managerial implications:}

The study offers suggestions for marketers and creators to leverage emotions to affect video-sharing behavior. This will help to increase the spread of the brand and contribute to the success of businesses marketing strategies.

Keywords: video-sharing, online video, on-sharing, viral video, viral marketing, emotion, and behavior. 


\section{INTRODUCTION}

Using social media to watch, post, and share videos has grown in popularity over the last decade, especially in the context of today's digital economy. YouTube is the world's most popular video-sharing network, with over 2 billion monthly unique users who watch over 1 billion hours of video per day and create billions of views. Mobile devices account for more than $70 \%$ of YouTube viewing time, and the platform reaches more users in the 18- to 49-year-old demographic than other social media platforms. (Statista, 2020; YouTube, 2020). The number of YouTube users and the average time spent per visit are both increasing, with the average mobile viewing session now lasting more than 40 minutes, up $50 \%$ in a year. (Statista, 2020; YouTube, 2020). Other social media platforms are seeing increases in video viewing, sharing, and uploading, with Facebook reporting significant increases in video content shared and seen, with over 64 billion video views each day. (Gahan, 2017)

Facebook is the largest social network in the world with 2.334 billion users, Facebook statistics show that $81 \%$ of businesses prefer to share their video content on Facebook, compared to other social media platforms. Users often use it to communicate, share and work. World-renowned brands, such as Berger King, Old Spice, and Unilever's Dove, have successfully employed videos to advertise in social media (Beverland et al., 2015) as a means to engage millions of consumers instantly for increasing brand awareness, for brand advocacy, and for generating a buzz (Harvey et al., 2011). For example, Ariel's "Share the Load" campaign, which challenges traditional gender roles, received 877,558 shares on social media, and 7-Eleven Thailand's tear-jerking Teachers' Day ad, which exemplifies the best of humanity, received 792,328 shares (Unruly, 2016), demonstrating the incredible reach and persuasiveness of videos for advertisements. As a result, marketers must pay attention to the factors that influence video sharing and spread, as well as take advantage of the social networking platform Facebook's sharing behavior to develop appropriate advertising strategies that will increase their ability to reach customers and promote their brands.

Previous studies have found that emotional arousal and emotional valence are important for video ads to be shared. As marketers are exploring new strategies to increase their viral effectiveness in social media, there is a need to provide guidelines on how to elicit emotional engagement that can trigger advertising virality. (Nikolinakou \& King, 2018). Bridget Rubenking (2019) investigated how user motives and emotional content of video messages evoking the aroused emotion of disgust influence an undergraduate student's willingness to share. The data also revealed some surprising findings, such as the fact that commercial video messages elicited higher levels of sharing intent than news and user-generated videos. According to functional theories of disgust, videos that triggered higher-order, socio-moral disgust were more likely to be shared than videos that elicited sexual or pathogen avoidance disgusts. (Rubenking, 2019). Prior studies also discovered what motivates individual online users to share their videos on YouTube and whether gender affects those motivations. The results show that positive emotions of amusement, excitement, inspiration, and warmth positively affect sharing. (Tellis et al., 2019; Yang et al., 2010). Researchers classified YouTube videos into six emotion categories and demonstrate sentiment analysis as a promising tool to quantify consumer responses towards branded video advertisements and thereupon, propose a sentiment-based typology of viral ad sharers. (Chen et al., 2017; Kulkarni et al., 2020). The recent studies investigated the way emotional appeals are being used in YouTube advertisements to promote products by considering various big brands of different industries and discovering attractive segments in the user-generated video streams as well as identify the time frames with the strongest display of emotion and rated the emotional intensity of each video(Chen et al., 2021; Wang et al., 2020). Scholars and practitioners are interested in studying emotions and online video-sharing behavior. The findings of these investigations, however, are inconsistent. There is evidence that positive emotions (e.g. joy) have the most impact on video-sharing behavior, but there is also evidence that negative emotions (e.g. disgust) have a more significant impact. As a result, further research on the impact of emotions on video-sharing behavior is necessary. Based on the Theory of Reasoned Action and emotion literature, the current research continues exploring the influence of emotions on the video-sharing behavior on Facebook of the young generation in a developing country. The findings will contribute to behavioral data in the emerging market.

The following is how the rest of the paper is organized: Section 2 discusses the research methodology, including the formulation of measurement items, sample characteristics, survey administration, data processing techniques, model research and hypothesis. Section 3 presents the results and discuss along with their implications. Finally, Section 4 mentions the contributions, concluding remarks, study limitations, and future research directions.

\section{METHODOLOGY}

This research is conducted in two phases. It includes preliminary research by qualitative method and formal research by quantitative method.

\subsection{Qualitative method}

For this study, qualitative research having the primary goal of adjusting and supplementing the research model, as well as completing and consolidating it. This study selected a sample with judgment and germination methods. Based on experience, the researcher selected the interviewee, and this person continued to introduce the next respondent. Aside from that, expert consultation aids in the revision of a questionnaire to 
investigate the elements influencing the intention of young people aged 15 to 24 to share videos. An in-depth interview with one (01) experienced expert with several years of expertise in Multimedia was done for this study. A focus group of seven (07) people aged 15 to 24 was also interviewed as part of the current investigation. They are current students and staff from various universities, with four persons being questioned face-to-face and two being interviewed via Google Meet Based on the Theory of Reasoned Action and emotion literature, and qualitative research results, the research model of this research is shown in Fig. 1.

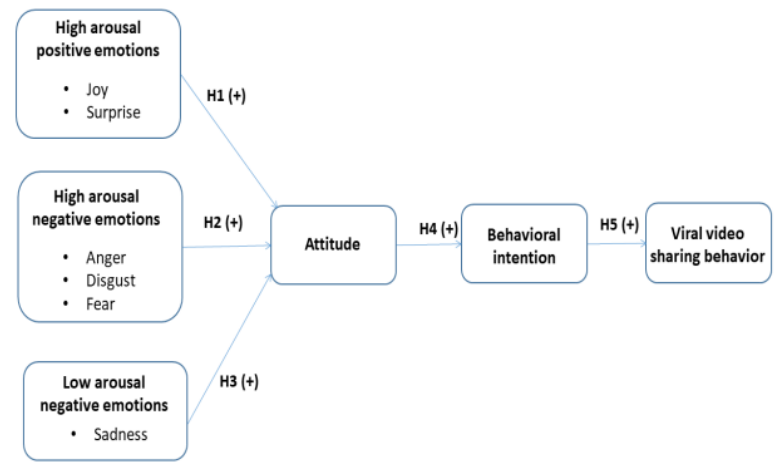

Fig. 1: Research model

H1: High arousal positive emotions (joy, surprise) positively influence attitude toward video sharing on Facebook.

H2: High arousal negative emotions (anger, disgust, fear) positively influence attitude toward video sharing on Facebook

H3: Low arousal negative emotions (sadness) positively influence attitude toward video sharing on Facebook.

H4: Attitude toward video sharing positively influences sharing behavioral intention.

H5: Behavioral intention positively influences video sharing behavior on Facebook

\subsection{Quantitative method}

We discovered during the data collection process that a set of questions did not ensure the separation of emotional elements, making it impossible for respondents to provide suitable responses. Furthermore, respondents will be required to watch all videos relevant to each emotion group to complete general study, which will be time consuming and may result in inaccurate responses. As a result, we decided to conduct four distinct experiments on the impact of each emotion group on video sharing behavior. Although the research model has only three groups of emotions, however, as analyzed in the proposed model design, negative emotions are easier to distinguish than positive emotions because they are very different. In addition, only the high arousal negative emotion group has three components (anger, disgust, fear), so we divided this emotion group into two smaller groups: (1) anger and (2) disgust/fear. Respondents watch videos corresponding to emotional groups and answer questions requested. Four separate studies are as below.

(1) High arousal positive emotions (joy and surprise) and video sharing behavior of Young Generation.

(2) High arousal negative emotions (anger) and video sharing behavior of Young Generation.

(3) High arousal negative emotions (disgust/fear) and video sharing behavior of Young Generation.

(4) Low arousal negative emotions (sadness) and video sharing behavior of Young Generation.

We calculated the sample size (n) using the known population size $(\mathrm{N}=11.891 .923)$ (General Statistics Office, 2019), the acceptable error value $(\mathrm{e}=0.05)$, and the following formula:

$$
n=\frac{\mathrm{N}}{1+\mathrm{N}\left(\mathrm{e}^{2}\right)}=400
$$

Because the research was subdivided into 4 other research so each research will have sample size $n=100$.

The current study has a maximum of 19 research questions for each small research. Use the formula: Sample size $=$ number of questions $\times 5=19 \times 5=95$, therefore $n=100$ is satisfied this requirement.

However, we did a sample size survey of 528 participants aged 15-24 with a study scope in Vietnam in order to conduct quantitative research. This age range was chosen because it represents the age group of young people who utilize social media in a widespread manner, such as Facebook. Furthermore, this age group is more likely to share material, particularly videos, on this social networking platform. To ensure that the projected sample size is satisfied, we employ the probability sampling method's cluster sampling approach to collect data from five important Vietnamese cities: HCMC, Hanoi, Danang, Hai Phong, and Can Tho.

Table 1: Sample size

\begin{tabular}{|c|c|c|}
\hline City & People aged $15-24 *$ & Percentage \\
\hline Ha Noi & $\underline{1,140,429}$ & $35.0 \%$ \\
\hline Hai Phong & 228,609 & $7.0 \%$ \\
\hline Danang & $\underline{195,753}$ & $6.0 \%$ \\
\hline Ho Chi Minh & $\underline{1,494,058}$ & $45.9 \%$ \\
\hline Can Tho & $\underline{198,124}$ & $6.1 \%$ \\
\hline Total & $3,256,973$ & $100 \%$ \\
\hline
\end{tabular}

* Source: General Statistics Office, 2019

The approach is indirect over the Internet based on judgment and convenience methods. The survey link (Google Form) was sent directly to the respondents via social networks such as Facebook Messenger, Zalo. We sent to 600 people and received 528 valuable responses.

\section{RESULTS AND DISCUSSION}

\subsection{Research 1}

This research conducted a survey with 123 people in the target population by conducting an online survey because of the epidemic situation. After collecting and 
testing, the research team put 123 encrypted copies into SPSS software for analysis.

Hanoi, Hai Phong, Danang, HCMC, and Can Tho have 35.0 percent, 7.3 percent, 5.7 percent, 45.5 percent, and 6.5 percent of respondents, respectively. This result can be regarded to be near to the aim, with cluster sampling of 35.01 percent Hanoi, 7.02 percent Hai Phong, 6.01 percent Danang, 45.87 percent Ho Chi Minh City, and 6.08 percent Can Tho. Males make up 45.5 percent of the population, while females make up 54.5 percent. Joy/surprise was chosen by 116 respondents as the emotion category that they shared the most, accounting for 94.3 percent of the total. In addition, 0.8 percent, 0.8 percent, and 4.1 percent of respondents indicated anger, disgust/fear, and sadness, respectively. The majority of respondents (52.0 percent) shared videos 1-2 times per week, with 46 people sharing videos 3-4 times per week accounting for 37.4 percent, 8 people sharing videos 5-6 times per week accounting for 6.5 percent, and 5 people sharing videos more than 6 times per week accounting for 4.1 percent.

The results suggest that the variables in the model are reliable, with Cronbach's Alphas of 0.868, 0.815, 0.759, and 0.842 for Emotion (EM1), Attitude (AT1), Behavioral intention (BI1), and Video-sharing behavior (SB1), respectively. Confirmatory Factor Analysis (CFA) has good result with Chi-square/DF (CMIN/DF) 1.736, GFI (Goodness-of-Fit Index) 0.930, CFI (Comparative Fit Index) 0.865, and RMSEA (Root mean square errors of approximation) 0.078 .

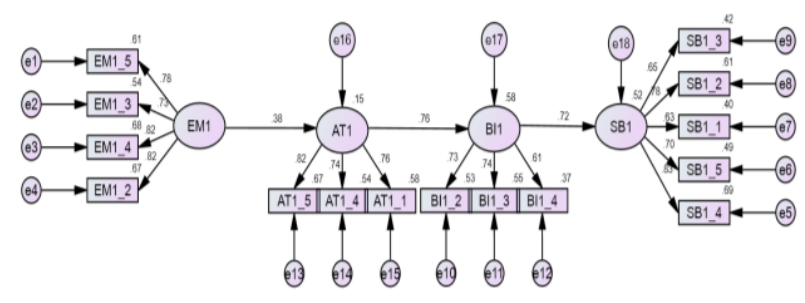

Fig. 2: Hypotheses testing result_1

Structural Equation Modeling Analysis shows that the regression weight of the relationship between EM1 and AT1 is 0.279, AT1 and BI1 is 0.803, BI1 and SB1 is 0.89 , with $\mathrm{P}$-values $<0.05$. Therefore, hypotheses $\mathrm{H} 1$, H4, H5 are supported.

The estimate values are used to evaluate the impact level of independent variables on dependent variables. In terms of significance, when EM1 increases by 1 standard deviation unit, AT1 increases by 0.383 standard deviation units, when AT1 increases by 1 standard deviation unit, BI1 increases by 0.760 standard deviation units, and when BI1 increases by 1 standard deviation unit, SB1 increases by 0.721 standard deviation units. Generally, the level of impact of SB1 on BI1 and AT1 on BI1 are high, but the level of impact of EM1 on AT1 is quite low.

Table 2: Regression Weights_1 and Standardized Regression Weights_1

\begin{tabular}{|c|c|c|c|c|c|c|c|}
\hline \multicolumn{8}{|c|}{ Regression Weights_1 } \\
\hline & & & Estimate & S.E. & C.R. & $\mathrm{P}$ & Label \\
\hline AT1 & $<---$ & EM1 & .279 & .078 & 3.599 & $* * *$ & \\
\hline BI1 & $<---$ & AT1 & .803 & .129 & 6.220 & $* * *$ & \\
\hline SB 1 & $<---$ & BI1 & .891 & .146 & 6.080 & $* * *$ & \\
\hline \multicolumn{8}{|c|}{ Standardized Regression Weights_1 } \\
\hline & & & \multicolumn{5}{|c|}{ Estimate } \\
\hline AT1 & $<---$ & EM1 & \multicolumn{5}{|c|}{.383} \\
\hline BI1 & $<---$ & AT1 & \multicolumn{5}{|c|}{.760} \\
\hline SB1 & $<--$ & BI1 & \multicolumn{5}{|c|}{.721} \\
\hline
\end{tabular}

Because the emotion group Joy/surprise has a low influence on attitude. As a result, in addition to creating videos with emotional elements, it is necessary to combine other factors to have a greater impact on attitudes. Marketers use video storytelling to influence the emotions and attitudes of viewers, causing them to remember a story and then their brand. Furthermore, sound, eye-catching images, and colors that stand out can be considered by marketers. Color has been shown in studies to influence viewers' emotions, physiology, and behavior. Sharpness is particularly evident in gender, as women are more sensitive to bright colors than men. Taking note of this, marketers can combine the aforementioned factors, as well as, of course, the emotional Joy/surprise element, for a video to truly touch the hearts of viewers, prompting them to share. Brand awareness will rise as a result of connection and interaction.

The standardized estimate (S.ES) demonstrates an indirect relationship between the Joy/surprise emotion group and extremely high sharing behavior. As a result, when creating a video, it is critical to focus on this group of emotions. Today, videos, particularly commercial videos, are frequently used by publishers. Making an emotional connection is an excellent complement to a great product. Thanks to this combination, customers will purchase the product without giving much thought to the price. Because the purchase decision is made from the heart and is the result of a perfect brand connection at that time. As a result, using emotional factors will help consumers have a more positive view of the brand. Find out what customers think of when they think of you. A good product is only the start of great service. The emotional appeal of the brand becomes the primary motivator for consumers to purchase. To win your customers' hearts, you must create emotional experiences that engage them with your brand. Of course, people appreciate it when a brand provides a certain level of value. Manufacturers 
and marketers will gain loyal audiences and customers as a result of this strategy.

\subsection{Research 2}

Because of the pandemic scenario, this study surveyed 132 people in the target population using an online survey. The research team entered 132 encrypted copies into SPSS software for analysis after collecting and testing.

The answer rates were 34.85 percent, 6.06 percent, 46.21 percent, and 6.06 percent in Hanoi, Hai Phong, Danang, HCMC, and Can Tho, respectively. With cluster sampling of 35.01 percent Hanoi, 7.02 percent Hai Phong, 6.01 percent Danang, 45.87 percent Ho Chi Minh City, and 6.08 percent Can Tho, this result can be considered close to the goal. Male responses make up 40.91 percent of the total, while females make up 59.09 percent.

Joy/surprise was chosen by 111 respondents as the emotion group that they shared the most, accounting for 84.09 percent of the total. In addition, 11.36 percent, 2.27 percent, and 2.27 percent of respondents chose anger, disgust/fear, and sadness, respectively. People who shared videos 1-2 times per week accounted for 88.64 percent of respondents, while those who shared videos 3-4 times per week accounted for 11.36 percent. Cronbach's alpha of all items is higher than 0.7. Corrected Item-Total Correlation of all items of emotion, attitude, behavioral intention, and sharing video sharing behavior variables are higher than 0.3 . The reliability of all item scales is acceptable. CFA model is fitted with Chi-square/DF (CMIN/DF) 1.665, GFI (Goodness-of-Fit Index) 0.876, CFI (Comparative Fit Index) 0.946, and RMSEA (Root mean square errors of approximation) 0.071 .

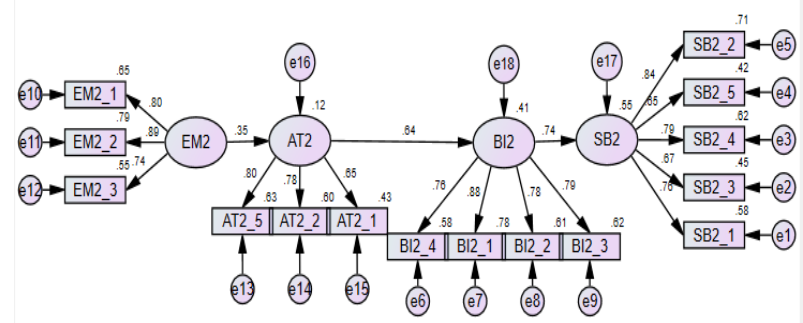

Fig. 3: Hypotheses testing result_2

The regression weight of the relationship between EM2 and AT2 is 0.316, between AT2 and BI2 is 0.771, and $\mathrm{BI} 2$ and SB2 is 0.704, all with P-values less than 0.05, according to Structural Equation Modeling Analysis. Hypotheses H2, H4, and $\mathrm{H} 5$ are supported.

SB2 has a high-level impact on BI2 and AT2 has a low-level impact on BI2, whereas EM2 has a low-level impact on AT2. When EM2 rises by one standard deviation unit, BI2 rises by 0.349 standard deviation units, AT2 rises by one standard deviation unit, BI2 rises by 0.643 standard deviation units, and SB2 rises by 0.743 standard deviation units when BI2 rises by one standard deviation unit. Generally, the level impact of $\mathrm{SB} 2$ on BI2 and $\mathrm{AT} 2$ on $\mathrm{BI} 2$ are high, but the level impact of EM2 on AT2 is quite low.

Table 3: Regression Weights_2 and Standardized
Regression Weights_2

\begin{tabular}{|c|c|c|c|c|c|c|c|}
\hline \multicolumn{8}{|c|}{ Regression Weights_2 } \\
\hline & & & Estimate & S.E. & C.R. & $\mathrm{P}$ & Label \\
\hline AT2 & $<---$ & EM2 & .316 & .094 & 3.352 & $* * *$ & \\
\hline BI2 & $<--$ & AT2 & .771 & .132 & 5.841 & $* * *$ & \\
\hline SB2 & $<---$ & $\mathrm{BI} 2$ & .704 & .101 & 6.946 & $* * *$ & \\
\hline \multicolumn{8}{|c|}{ Standardized Regression Weights_2 } \\
\hline & & & \multicolumn{5}{|c|}{ Estimate } \\
\hline AT2 & $<---$ & EM2 & \multicolumn{5}{|c|}{.349} \\
\hline BI2 & $<---$ & AT2 & \multicolumn{5}{|c|}{.643} \\
\hline SB2 & $<--$ & BI2 & \multicolumn{5}{|c|}{.743} \\
\hline
\end{tabular}

\subsection{Research 3}

Research 3 collected 120 valuable responses. Hanoi, Hai Phong, Danang, HCMC, and Can Tho have 35.1 percent, 7.5 percent, 5.8 percent, 45.8 percent, and 5.8 percent of respondents, respectively. Males make for 46.7 percent of respondents, while females account for 53.3 percent. Joy/surprise was chosen by 92 respondents as the emotion category that they shared the most, accounting for 76.7 percent of the total. In addition, 8.3 percent, 6.7 percent, and 8.3 percent of respondents chose anger, disgust/fear, and sadness, respectively. 73.4 percent of respondents said they shared videos once to twice each week. People who shared video three to four times per week make up 25.0 percent, those who share video five to six times per week make up 0.8 percent, and those who share video more than six times per week make up 0.8 percent.

Cronbach's alpha of all items is higher than 0.8 showing that the scales have high reliability. All indexes of CFA meet the requirements.

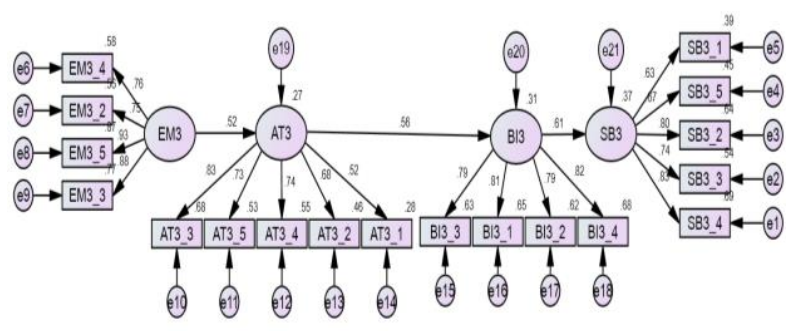

Fig. 4: Hypotheses testing result_3

Findings found that the regression weight of the relationship between EM 3and AT3 is 0.755, AT3 and $\mathrm{BI} 3$ is $0.634, \mathrm{BI} 3$ and SB3 is 0.486 , with $\mathrm{P}$-values $<0.05$. Therefore, hypotheses H2, H4, H5 are supported. The level impact of EM3 on AT3, AT3 on BI3, SB3 on BI3 varies from 0.522 to 0.611 . 
Table 4: Regression Weights_3 and Standardized Regression Weights_3

\begin{tabular}{|c|c|c|c|c|c|c|c|}
\hline \multicolumn{8}{|c|}{ Regression Weights_3 } \\
\hline & & & Estimate & S.E. & C.R. & $\mathrm{P}$ & Label \\
\hline AT3 & $\begin{array}{ll}<--- \\
-1\end{array}$ & EM3 & .755 & .150 & 5.016 & $* * *$ & \\
\hline BI3 & $<---$ & AT3 & .634 & .120 & 5.292 & $* * *$ & \\
\hline SB3 & $<--$ & $\mathrm{BI} 3$ & .486 & .083 & 5.867 & $* * *$ & \\
\hline \multicolumn{8}{|c|}{ Standardized Regression Weights_3 } \\
\hline & & & \multicolumn{5}{|c|}{ Estimate } \\
\hline AT3 & <--- & EM3 & \multicolumn{5}{|c|}{.522} \\
\hline $\mathrm{BI} 3$ & $\begin{array}{ll}<-- \\
\end{array}$ & AT3 & \multicolumn{5}{|c|}{.560} \\
\hline SB3 & $\begin{array}{ll}<--- \\
\end{array}$ & $\mathrm{BI} 3$ & \multicolumn{5}{|c|}{.611} \\
\hline
\end{tabular}

According to the findings of research 2 and research 3, the effect of anger, disgust, and fear on attitude towards sharing are not very high, but the attitude has a high impact on behavioral intention and behavioral intention has a high impact on video sharing behavior. Thus, administrators also need to focus on videos containing anger because it will lead to strong sharing behavior. Marketers should be concerned with promotional content that generates stronger angry reactions because it has the potential to spread a video quickly.

Brand video producers need to focus more on the content that arouses anger/ disgust/fear and bring it to climax, combined with the use of other factors (trends of the times, current issues, topical issues, etc.) because this will have a stronger influence on attitudes than just using emotional factors. However, the emotional factor still plays the most important role. In addition, the standardized estimate (S.ES) of the indirect relationship from anger/ disgust/ fear to video-sharing behavior is quite high, we see that the impact of direct anger on sharing behavior is quite strong, so, it is necessary to focus on this group of emotions when creating videos. Therefore, when designing a video for a product promotion strategy, it is necessary to pay attention to how the video brings emotions of anger, disgust, and fear to the customer, whether that emotion has a strong impact on making viewers share it immediately.

However, a shortcoming here is that very few commercial videos use the above emotions as primary emotions to spread, because overusing anger/ disgust/ fear can cause the brand to lose its image in the eyes of viewers and consumers. Sometimes it also has the opposite effect on customers' attitudes towards the brand image... Therefore, when creating videos for commercial purposes, marketers need to consider the appropriate interweaving of these emotions, enough to hit the psyche without causing discomfort to customers.
In addition, creating an anger/disgust/fear video is also quite difficult to combine with the trend element - an essential element to help the video go viral, so when catching the indignant and pressing issues of the moment, marketers need to release the video at the right time, the video doesn't need to be too picky about the script, but hitting the emerging trend can still create good effects on social networks. Marketers who grasp hot issues on social networks that cause disgust/fear need to release that video content at the right time, hitting the current trend will create a good viral effect on social networks.

\subsection{Research 4}

In Research 4, 153 participants from the target population were polled. The percentage of responders in Can Tho, Danang, Hai Phong, Hanoi, and HCMC was $5.9 \%, 7.2$ percent, 35.3 percent, and $45.8 \%$, respectively. Females make up 44.4 percent of the population, while males make up 55.6 percent. 92.8 percent of respondents identified joy/surprise as the emotion category with which they shared the most. As can be observed, the most typically shared emotion group is joy/surprise. The majority of respondents $(88.2 \%)$ said they shared videos 1-2 times each week. People who shared video 3-4 times per week made up 11.11 percent of the total, while those who shared 5-6 times per week made up 0.7 percent.

The reliability of all item scales and Confirmatory Factor Analysis are acceptable.

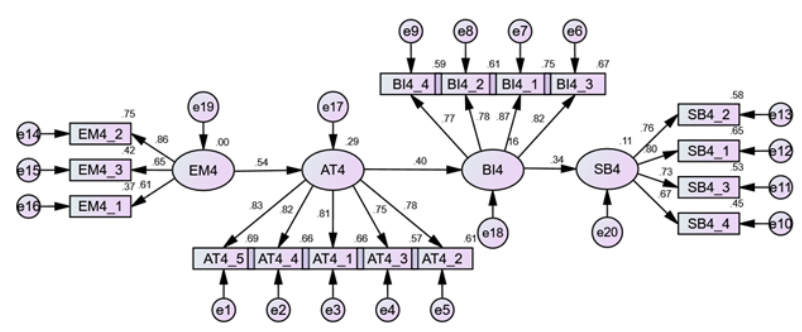

Fig. 5: Hypotheses testing result 4

Findings found that the regression weight of the relationship between EM4 and AT4 is 0.702, AT4 and BI4 is $0.634, \mathrm{BI} 4$ and SB4 is 0.224 , with P-values $<0.05$. Therefore, hypotheses H3, H4, H5 are supported. Consider estimate values, the level impact of EM4 on AT4 is pretty high, but the level impact of AT4 on BI4 and BI4 on SB4 is quite low. In terms of significance, when EM4 increases by 1 standard deviation unit, BI4 increases by 0.514 standard deviation units, when AT4 increases by 1 standard deviation unit, BI4 increases by 0.399 standard deviation units, and when BI4 increases by 1 standard deviation unit, SB4 increases by 0.337 standard deviation units.

Table 5: Regression Weights_4 and Standardized Regression Weights_4

\begin{tabular}{|c|c|c|c|c|c|}
\hline \multicolumn{6}{|c|}{ Regression Weights_4 } \\
\hline & Estimate & S.E. & C.R. & $\mathrm{P}$ & Label \\
\hline $\begin{array}{|lll|}\text { AT4 } & <--- & \text { EM4 }\end{array}$ & .702 & .132 & 5.335 & **** & \\
\hline
\end{tabular}




\begin{tabular}{|lll|llll|}
\hline BI4 & $<---$ & AT4 & .369 & .083 & 4.434 & $* * *$ \\
SB4 & $<---$ & BI4 & .224 & .065 & 3.470 & $* * *$ \\
& & & & & \\
\hline \multicolumn{5}{|c|}{ Standardized Regression Weights_4 } \\
\hline & & & Estimate \\
\hline AT4 & $<---$ & EM4 & .541 \\
\hline BI4 & $<---$ & AT4 & .399 \\
\hline SB4 & $<---$ & BI4 & \\
\hline
\end{tabular}

Unlike studies on strong emotional stimulation groups, the impact of sadness on video sharing behavior on the social networking platform Facebook is strong. On the contrary, the impact of attitude on intended behavior as well as intended behavior on video sharing behavior is not high. By referring to the table of results above, we can see that the impact coefficient (referred to as SES) of the relationship from sadness to behavior is quite low, the level is almost neutral. Videos with a simple sad feeling will be difficult to make viewers perform the sharing behavior. However, a video that carries a sad emotion like touching someone else's pain, will have a strong impact on their psyche, thereby increasing the intention to share that video.

But in reality, it is not easy to do this because it requires the video producer to have enough experience as well as a breakthrough initiative in content so that the new video will spread quickly and achieve success from the requirements of the business or the administrator. On the contrary, if done poorly, it will backfire, preventing it from spreading, making viewers feel bored, thereby not having a good impression of the brand. In short, videos with sad emotions don't have the same viral effect as strong emotional groups. But if administrators pursue this video genre, they need to build strong and in-depth content.

As mentioned above, sadness is a weak emotional stimulation group, its impact on young people's video sharing behavior on the social networking platform Facebook is low. Currently, the reality is that although there are many videos with this kind of emotion, the number of videos that can go viral is quite small. According to research, sad videos that can go viral are mostly videos about the pain of families, of women being discriminated against, or videos of separated love. It is rare to see videos promoting brands with sad emotions that can go viral. Most brands will use strong positive emotions such as joy and surprise to spread the message about their brand.

However, if administrators still want to use this emotion in videos, it may still be possible. Because the level of impact of this emotional group is quite low, the content must be carefully invested, embedding emotions into the viewer's basic pain such as unfair treatment, compassion, physical and mental sadness are strong enough to affect the psychology of viewers, thereby motivating them to share this video. Along with that are the refinement and good investment in terms of images and sound. That will be the viewer's first impression of this video. Colors in sad videos should use cold colors to make emotions easier to exploit. Properly and fully combining the above requirements, surely the communication effect of videos with sad emotions will achieve optimal efficiency for administrators and businesses.

\section{CONCLUSION}

This research has built a research model and scales on how emotions affect the behavior of sharing videos on the social networking platform Facebook of young people aged 15-24. Besides, we also tested and measured the factors built into the model. As expected, emotions positively indirectly affect young people's behavior of sharing videos on Facebook through attitude toward video sharing and behavioral intention. Positive strong emotions (joy, surprise), negative strong emotions (anger, disgust, fear), and negative weak emotions (sadness) all had a positive effect on video sharing behavior on Facebook of the young generation. However, the magnitude of their impact is not the same. Specifically, according to the analysis and data processing, the group of strong positive emotions and strong negative emotions strongly influenced the behavior of sharing videos more than the group of weak negative emotions. Other variables such as attitude, behavioral intention also have a positive impact on the performance of the final video sharing behavior. In general, emotion is a factor that administrators and businesses in the field of communication and viral marketing need to pay attention to promote video sharing behavior. On that basis, we propose some recommendations to businesses in this field and some methods to maximize the emotional element contained in videos posted on Facebook. From there, these videos can attract maximum views and shares, so it will make good communication as well as bring benefits to administrators and businesses.

\section{ACKNOWLEDGMENTS}

The authors gratefully acknowledge the contribution of students who have studied at School of International Business - Marketing, University of Economic Ho Chi Minh City, Ho Chi Minh, Vietnam.

\section{REFERENCES}

[1] Beverland, M., Dobele, A., \& Farrelly, F. (2015). The viral marketing metaphor explored through Vegemite. Marketing Intelligence \& Planning.

[2] Chen, H., Chin, K. L., \& Tan, C. B. (2021). Selection and validation of emotional videos: Dataset of professional and 
amateur videos that elicit basic emotions. Data in Brief, 34, 106662.

[3] Chen, Y.-L., Chang, C.-L., \& Yeh, C.-S. (2017). Emotion classification of YouTube videos. Decision support systems, 101, 40-50.

[4] Gahan, B. (2017). How Facebook Watch will overtake YouTube as the biggest video platform. Mashable. Retrieved September from

https://mashable.com/2017/12/05/how-fac ebook-watch-will-overtake-youtube-as-big gest-video-platform/

[5] General Statistics Office. 2019. Results of the 2019 Vietnam Population and Housing Census. 239-309.

[6] Harvey, C. G., Stewart, D. B., \& Ewing, M. T. (2011). Forward or delete: What drives peer-to-peer message propagation across social networks? Journal of Consumer Behaviour, 10(6), 365-372.

[7] Kulkarni, K. K., Kalro, A. D., Sharma, D., \& Sharma, P. (2020). A typology of viral ad sharers using sentiment analysis. Journal of Retailing and Consumer Services, 53.

[8] Nikolinakou, A., \& King, K. W. (2018). Video ads: Emotional triggers and social media virality. Psychology \& Marketing, 35(10), 715-726.

[9] Rubenking, B. (2019). Emotion, attitudes, norms and sources: Exploring sharing intent of disgusting online videos. Computers in Human Behavior, 96, 63-71.

[10] Statista. (2020). YouTube - Statistics \& Facts. Retrieved September, 2020 from https://www.statista.com/topics/2019/yout ube/

[11] Tellis, G. J., MacInnis, D. J., Tirunillai, S., \& Zhang, Y. (2019). What drives virality (sharing) of online digital content? The critical role of information, emotion, and brand prominence. Journal of Marketing, 83(4), 1-20.

[12] Wang, Z., Zhou, J., Ma, J., Li, J., Ai, J., \& Yang, Y. (2020). Discovering attractive segments in the user-generated video streams. Information Processing \& Management, 57(1), 102130.

[13] Yang, C., Hsu, Y.-C., \& Tan, S. (2010). Predicting the determinants of users' intentions for using YouTube to share video: moderating gender effects. Cyberpsychology, Behavior, and Social Networking, 13(2), 141-152.

[14] YouTube. (2020). YouTube by Numbers https://www.youtube.com/about/press/ 